\title{
Criação de um mapa de taxa volumétrica de calor radiogênico para a área de Vazante (MG) através de gamaespectrometria .
}

Jean Vinícius de Oliveira Lima ${ }^{1}$, Elton Luiz Dantas ${ }^{2}$, Débora Rabelo Matos ${ }^{1}$

${ }^{1}$ Instituto de Geociências, Universidade de Brasília, Brasília, Brasil

${ }^{2}$ Laboratório de Geocronologia, Instituto de Geociências, Universidade de Brasília, Brasília, Brasil

Copyright 2014, SBGf - Sociedade Brasileira de Geofísica.

Este texto foi preparado para a apresentação no VI Simpósio Brasileiro de Geofísica, Porto Alegre, 14 a 16 de outubro de 2014. Seu conteúdo foi revisado pelo Comitê Técnico do VI SimBGf, mas não necessariamente representa a opinião da SBGf ou de seus associados. É proibida a reprodução total ou parcial deste material para propósitos comerciais sem prévia autorização da SBGf.

\section{Abstract}

We developed an algaritime that calculate in a Geosoft a map of the distribution of radiogenic heat production in the Brasilia fold thrust belt. We chose an area in the Vazante the region, Minas Gerais.

The data derived from the concentration of radionuclides by gamma spectrometry mesure, using the methodology suggested by Ryabach (1976) and Bucker and Ryabach (1996). We use data collected by airbone gamma ray spectrometry survey. Our results suggest a lateral variation of the rate of heat flow in the RHP and the temperature of the upper crust. This map revealed a strong RHP variation in the Vazante region (between 0.36 and $\left.6.20 \mu \mathrm{W} / \mathrm{m}^{3}\right)$.

\section{Introdução}

A produção de calor radiogênico em rochas é normalmente atribuída à relação entre a densidade e a concentração dos elementos potássio (K), tório (Th) e urânio (U) em rochas, medida com a ajuda de gamaespectrômetros. A contagem de radiação gama total (CT) é gerada devido à presença destes três elementos, e representa em torno de dois terços de todo calor radiogênico da terra. Através de dados radiométricos é possível gerar mapas de calor radiogênico para diferentes compartimentos da litosfera (Bodorkos et al., 2004; Rybach ,1986).

Neste trabalho se dispõe a desenvolver a automação do cálculo da taxa de calor em ambiente Geosoft, utilizando o método definido por Bücker e Ryabach (1996), segundo o qual se relaciona linearmente a produção de calor radiogênico com a contagem de radiação gama total em unidades API (American Petroleum Institute).

A área de estudo é na região próxima a Mina de Morro Agudo, MG, que contém um depósito de Au e um depósito de $\mathrm{Zn}$ sulfetado na mina de Vazante.

\section{Determinação da produção de calor}

Quatro radioisótopos, $\mathrm{U}^{238}, \mathrm{U}^{235} \mathrm{e} \mathrm{Th}^{232}$ e $\mathrm{K}^{40}$, ocorrem em abundância suficiente para contribuir para o orçamento térmico da litosfera; estes elementos são referidos como elementos produtores de calor radiogênico (Sapucaia et al, 2005). 
Segundo a fórmula de Ryabach (1986), para uma amostra de rocha de densidade $\rho$ $\left(\mathrm{Kg} / \mathrm{m}^{3}\right)$, a taxa volumétrica de calor radiogênico (A) é dado por:

$$
A\left(\mu W / m^{3}\right)=10^{-5} \rho\left(9,52 C_{U}+2,56 C_{T h}+3,48 C_{K}\right)
$$

Onde $C_{U}$ e $C_{T h}$ são respectivamente a concentração em ppm de U e de Th e $C_{K}$ é a concentração em porcentagem de K.

$\mathrm{O}$ registro que mede a contagem de radioatividade de gama total (CT) é dado pela soma da contribuição radioativa do $\mathrm{U}$ (principalmente pela emissão de raios gama do elemento-filho $\mathrm{Bi}^{214}$ ), do $\mathrm{Th}$ (principalmente pelo elemento-filho $\mathrm{Tl}^{208}$ ) e pelo $\mathrm{K}^{40}$.

Segundo Bücker e Ryabach (1996) há uma relação linear entre a produção de calor e a contagem de radioatividade total dada por:

$$
A\left(\mu W / m^{3}\right)=0,0158(C T(A P I)-0,8)
$$

Onde a contagem de radioatividade total é dada em unidade API.

Sabe-se apenas que esta depende principalmente da litologia e que decresce com a profundidade, ainda que de modo não sistemático em regiões metamórficas (Ashwal et al.,1987).

Em uma bacia sedimentar, parcelas do calor radiogênico são principalmente produzidas pelas rochas do embasamento, bem como pelas camadas sedimentares da própria bacia (estas menos importantes). Também tem significativa importância, a soma do calor proveniente de fontes mantélicas (astenosfera) aos demais compartimentos para definir a história térmica da bacia. No caso de prospecção de petróleo de uma bacia, o conhecimento de sua história térmica é importante, pois dará indicações se esta foi capaz de gerar depósitos minerais ou hidrocarbonetos.

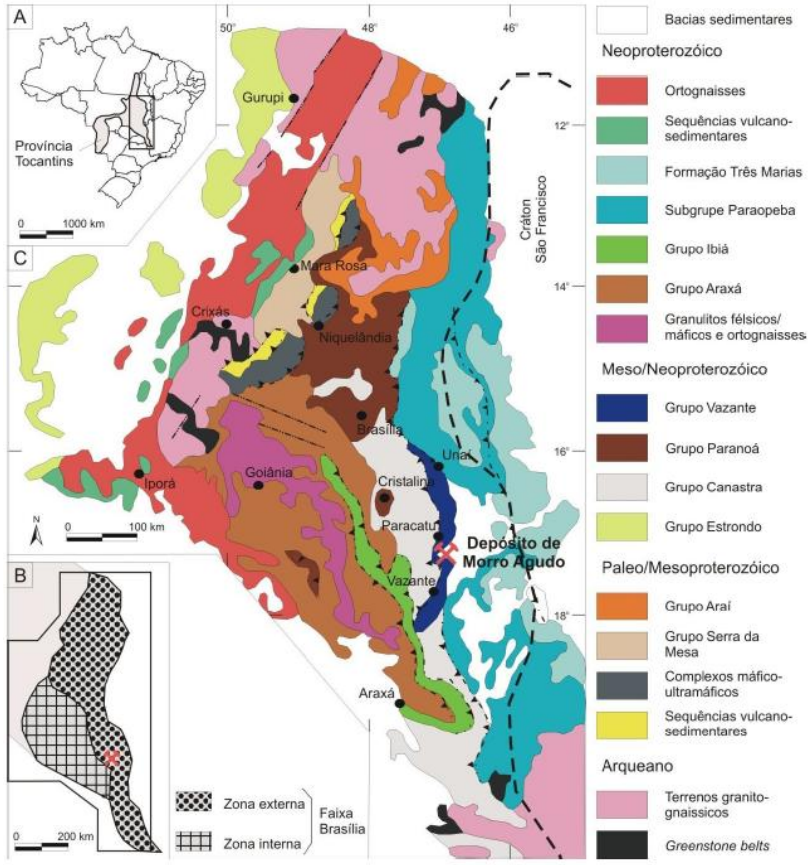

Figura 1: A)Localização de Província Tocantins no território brasileiro. B) Detalhe da referida província enfocando a faixa Brasília e sua Zonas Interna e Externa (onde se localiza o depósito de Morro Agudo). C) Mapa geológico regional da Faixa Brasília.

Em ambientes geradores de depósitos minerais, o transporte de materiais enriquecidos em urânio, tório e potássio ocorre através de atuações de diversas fontes, tais como, processos metamórficos, de fusão crustal, metassomatismo e hidrotermalismo. A distribuição destes elementos radioativos nas rochas está diretamente ligada a estes processos, que normalmente ocorrem em diferentes profundidades na crosta terrestre e com variação na escala do tempo. A diferenciação magmática é responsável pela distribuição inicial destes mesmos radioelementos sendo os dois últimos mais sensíveis aos vários processos dessa diferenciação (Adams \& Gasparini, 1970). A atuação posterior de processos metamórficos altera a distribuição destes elementos enriquecendo alguns de seus níveis. Da mesma maneira, o hidrotermalismo tende a redistribuir estes elementos, trazendo-os para as porções mais externas da crosta.

\section{Geologia regional da área de estudo}

A área enfocada neste trabalho contém um depósito de $\mathrm{Zn}$ sulfetado (sphalerite-rich carbonatehosted $\mathrm{Zn}-(\mathrm{Pb}))$, conhecido como 
depósito de Fagundes, e está inserida no mais importante distrito rico em $\mathrm{Zn}$ do Brasil (Monteiro et al. 2006). Este depósito é caracterizado como do tipo MVT (Mississipi Valley-Type) e ocorre associado às rochas carbonáticas da Formação Morro do Calcário (Figura 1).

Sobreposta a essa formação, ocorrem pelitos, com dolomitos, conglomerados e arenitos subordinados, cartografados como Formação Lapa. Essas formações pertencem ao Grupo Vazante, de idade Neoproterozóica, cuja estratigrafia formalmente estabelecida data do final da década de 90 (Dardenne et al. 1998 e Dardenne 2000). A região compreende o maior distrito de zinco do país no qual estão inseridos os depósitos de $\mathrm{Zn}-\mathrm{Pb}$ de Morro Agudo, Vazante, Ambrósia e Fagundes.

O Grupo Vazante compreende rochas carbonáticas e pelitos metamorfizados em fácies xisto-verde que ocorrem com uma faixa $\mathrm{N}-\mathrm{S}$ de $250 \mathrm{~km}$ de extensão na porção leste de Faixa Brasília. Dardenne (2000) argumenta que o contexto tectônico do grupo indica uma sedimentação anômala em uma zona rápida de subsidência. Com isso a ocorrência de carbonatos plataformais de águas rasas em estromatólitos, a ausência de camadas de cinzas vulcânicas (Dardenne, 2000) e idades Sm-Nd sugerem sedimentação do Grupo Vazante em margem passiva (Dardenne, 2000; Pimentel et al., 2001; Azmy et al., 2008).

\section{Processamento}

Os dados gamaespectrométricos foram coletados na Área 1 Unaí-Paracatu-VazanteCoromandel de Minas Gerais maior, durante o curso dos levantamentos geofísicos aéreos conduzidos pela SEME (Secretaria do Estado de Minas e Energia do Governo de Minas Gerais ) e realizados pela LASA Engenharia e Prospecções S.A. Linhas de pesquisa foram levados N30W, com espaçamento de $250 \mathrm{~m}$, e foram amarrados por linhas N50E a $2500 \mathrm{~km}$ de espaçamento. A altura média de voo foi de $100 \mathrm{~m}$ com uma velocidade média de 200 $\mathrm{km} / \mathrm{h}$. Os espectros de raios gama foram amostrados a intervalos de 1 segundo, utilizando um gamaespectrômetro multicanal Picodas, modelo PGAM-1000 e um sistema de navegação GPS Picodas/Novatel de 12 canais. Acima da faixa de energia $0-3 \mathrm{MeV}$, 256 canais de dados foram coletados e os cinco de energia seguintes janelas foram monitorados constantemente: contagem total $(0,41-2,81 \mathrm{MeV})$, potássio $(1,37-1,57 \mathrm{MeV})$, urânio $(1,66-1,86 \mathrm{MeV})$, tório $(2,41-2,81$ $\mathrm{MeV})$ e da radiação cósmica (3,00-6,00 $\mathrm{MeV})$.

Após a calibração do detector de energia e as correções de radiação cósmica e atmosférica, os espectros de raios gama foram somados para as janelas de energia descritas acima.

As taxas de contagem de janelas foram retiradas (correção de interação de canal devido ao efeito Compton) e correção de desvios na altura aeronaves da altura de voo nominal. Os dados foram corrigidos e então gridados com um tamanho de célula de $60 \mathrm{~m}$, utilizando um algoritmo de mínima curvatura. A construção do mapa de taxa de produção de calor foi através dos dados de contagem de radioatividade de gama total (CT). Para isto, criou-se o canal da taxa de produção de calor radiogênico (A), onde foi utilizada equação de Bücker e Ryabach (1996) no canal CT para chegar aos valores de A. No entanto, para se usar esta equação necessita-se que os valores de CT estejam em unidades API e os valores estavam no banco de dados em $\mu \mathrm{R} / \mathrm{h}$.

Hilchie (1979) comprovou que a conversão de $\mu \mathrm{R} / \mathrm{h}$ para API depende muito das características do gamaespectrômetro utilizado. Segundo este mesmo autor, um valor médio seria assumir que $1 \mu \mathrm{R} / \mathrm{h}$ equivale de 15 API. Analisando-se a fórmula de Bücker e Ryabach (1996) se pode ver que a relação ente CT e A é linear, por isso também se gridou CT.

Neste trabalho foi feito um procedimento que calcula o fluxo de calor a partir dos dados de 
gamaespectrometria de levantamentos aerogeofísicos.

\section{Resultado e discussão de dados}

Os produtos mais relevantes gerados após o processamento foram os mapas de concentração ternário (RGB), A (API), e CT que seriam respectivamente as Figuras 2, 3, 4. Nestes mapas se pode observar que domínios radiométricos estão extremamente correlacionados as taxas de fluxo de calor radiogênico.

Nas figuras, os pontos mais claros, com maior concentração de $\mathrm{K}$, Th e U do mapa ternário, (Figura 6) coincidiram com as regiões de maior taxa de calor (Figura 7). De forma análoga os pontos com menor presença de $\mathrm{K}$, Th e U foram onde se observou as menores taxas de produção de calor radiogênico.

Os mapas de A (Figura 3) e de CT (Figura 4), em geral, possuem anomalias muitos semelhantes que se distinguem em termos de unidade de medida e amplitude: Enquanto a produção de calor (A) variou de 0,36 a 6,20 $\mu \mathrm{W} / \mathrm{m}^{3}$ CT variou de 1,84 a $34,07 \mu \mathrm{R} / \mathrm{h}$. A produção de calor radiogênico está intimamente ligada com o canal de contagem total, mas é válido lembrar que os valores medidos são somente dois terços do fluxo de calor radiogênico da região.

\section{Conclusão}

Pôde-se ver empiricamente que há uma relação muito forte entre a contagem total de radiação e a taxa volumétrica de calor radiogênica calculada segundo a fórmula de Bücker e Ryabach (1996). É visto nos mapas CT (contagem total) e A (taxa volumétrica de calor) que as anomalias de taxa de calor possuem formas semelhantes ao mapa de contagem total diferenciando somente em termos de unidade e magnitude.

Logo, além de indicar a variação radiométrica espacial, se pode dizer que este dado de CT representa o fluxo de calor radiogênico de uma determinada área.

\section{Bibliografia}

Bücker, C.; Rybach, L. A simple method to determine heat production from gamma-ray logs. Elsevier Science Publishers, v 13, n.4, p.373-375, 1996.

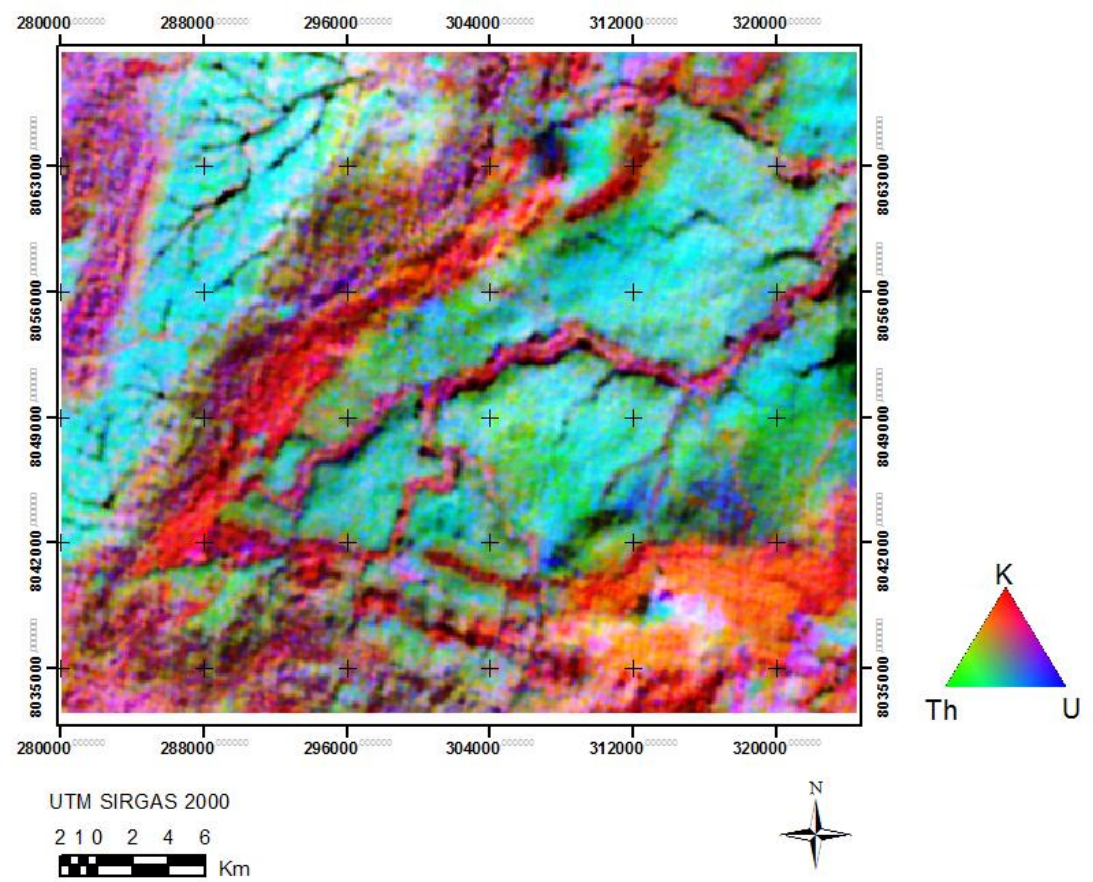




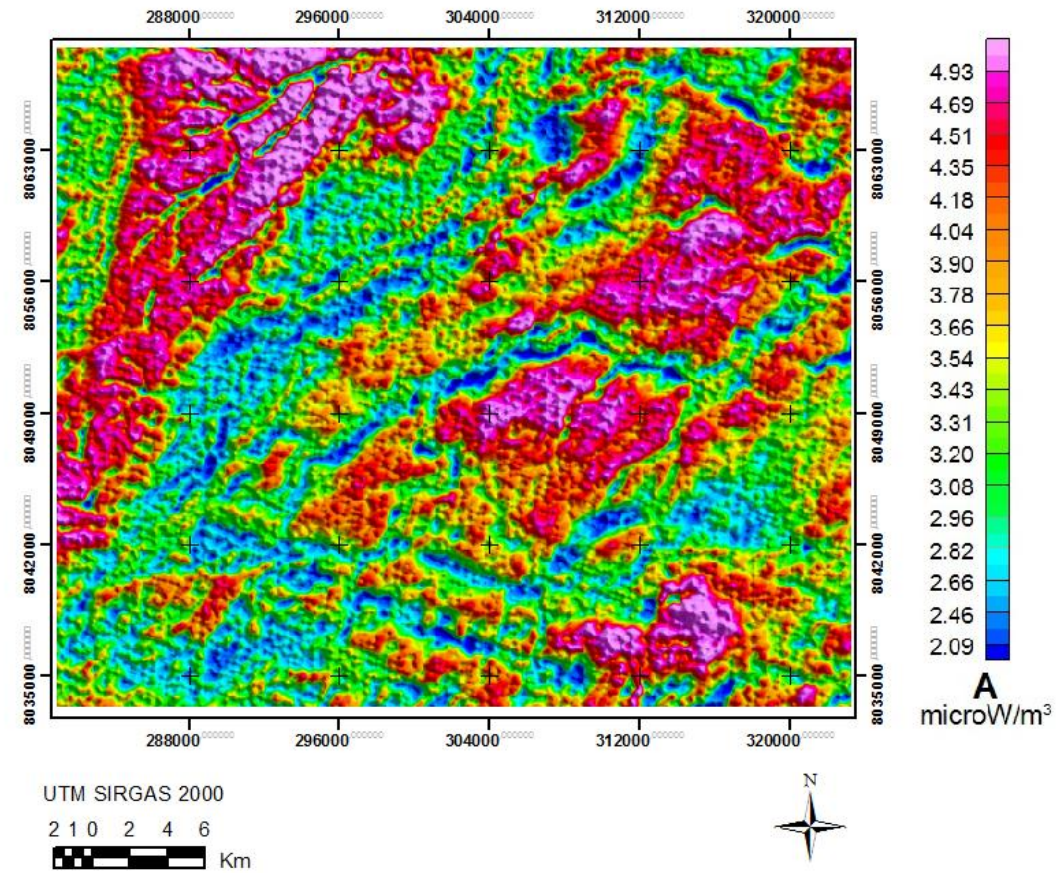

Figura 3: Mapa de A (taxa volumétrica de produção de calor radiogênico em $\mu \mathrm{W} / \mathrm{m}^{3}$ ).

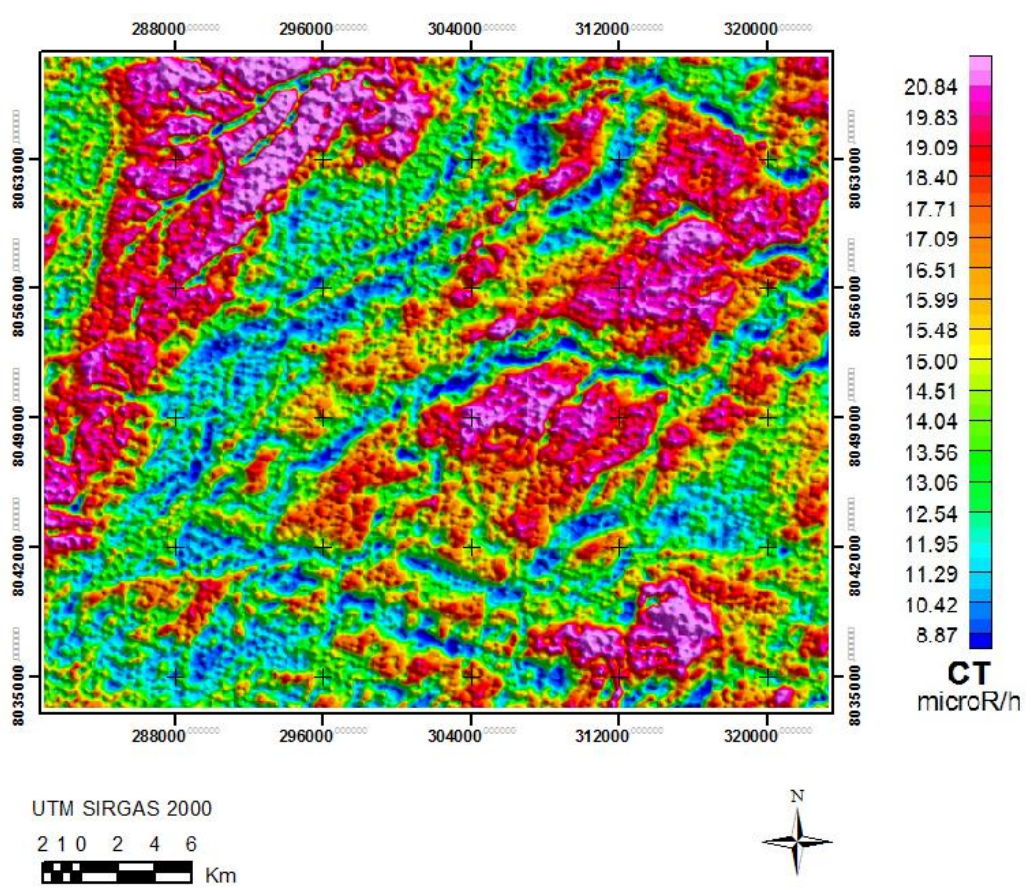

Figura 4: Mapa CT (contagem total em $\mu \mathrm{R} / \mathrm{h}$ ).

Kukkonen, I.T. Terrestrial het flow and radiogenic heat production in Finland, the central Baltic Shield. Elsevier Science Publishers - Tectonophysics, p. 219-230, 1989.

Bodorkos, S.; Sandiford, M; Minty, B.R.S; Blewett, R.S. A high-resolution, calibrated airbone radiometric dataset applied to the estimation of crustal heat production in the Archean northern Pilbara Craton, Weastern
Australia. Elsevier Science Publishers Precambrian Research 128, p. 57-82, 2004.

Sapucaia, N.S.; Argollo, R.M.; Barbosa, J.S.F. Teores de potássio, urânio, tório e taxa de produção de calor radiogênico no embasamento adjacente às bacias sedimentares de Camamu e Almada, Bahia, Brasil. Revista Brasileira de Geofísica, v. 23, p. 453-475, 2005.

Dardenne, M.A.; Freitas-Silvas, F.H. Pb-Zn ore deposits of Bambui and Vazante groups, 
in São Francisco Craton and Brasilia Fold

Belt, Brazil. Base metal Deposits of Brazil, p.

76-83, 2000.

Santana A.V.A. Estratigrafia, sedimentologia e proveniência das unidades superiores do Grupo Vazante na região da Fazenda Fagundes, Paracatu. Dissertação de Mestrado n²83, Instituto de Geociências - UnB, 2011

Neves, L.P. Características descritivas e genéticas do Depósito de Zn-Pb Morro Agudo, Grupo Vazante. Dissertação de Mestrado n $^{\circ} 280$, Instituto de Geociências UnB, 2011.

Hilchie, D.W. Old Electrical Log Interpretation Tulsa, Oklahoma, Chapter 10, p. 137-147, 1979.

Adams, J; Gasparini P.; Gamma-ray spectrometry of rocks (Methods in geochemistry and geophysics Series). Elsevier Publishing Company, 1970.

Monteiro, L.V.S.; Bettencourt, J.S.; Juliani, C.; Oliveira, T.F. Geology, petrography and mineral chemistry of Vazante, Ambrásia and Fagundes Neoproterozoic.

Ashwal,L. D.; Morgan, P.; Kelley, S. A.; Perciva, J. A. Heat production in an Archean crustal profile andimplications for heat flow and mobilization of heatproductingelements. Earth and Planetary Sciences Letters, Amsterdam, v. 85, n. 4, p. 439-450, 1987.

Argollo, R.M.; Marinho, M.M.; Costa, A.B.; Filho, H.A.S.; Santos, E.J.; Coutinho, L.F.C. Modelo crustal e fluxo de calor nos domínios Estância, Canudos-Vaza-Barris e Macururé adjacentes às bordas emersas da Bacia Sergipe-Alagoas. B. Geoci. Petrobras, RJ, v. 20, n. 1/2, p. 283-304, nov.2011/nov.2012. 